\title{
Behavioral heat intake as a function of reward duration in rats
}

\author{
ROBERTO REFINETTI and HARRY J. CARLISLE \\ University of California, Santa Barbara, California
}

\begin{abstract}
Animals working for heat in a cold environment increase responding when reward duration is reduced, but in many instances the increase in responding is not sufficient to prevent a decrease in the amount of heat obtained. Eight experiments were conducted to investigate the reason why rats' barpress responses for heat are less efficient at short reward durations. The results show that a ceiling effect is not involved and also that improper response-topography or differences in whole-body heat absorptivity or in response effort cannot explain the phenomenon. Evidence was found for the hypothesis that response rate does not increase sufficiently at short reward durations because many short rewards produce a greater afferent signal than do few long rewards. This inequality seems to be caused by characteristics of the stimuli that result in a greater relative change in skin temperature $(\Delta \mathrm{T})$ or in the rate of change of skin temperature $(\delta \mathrm{T} / \delta \mathrm{t})$ for short-duration rewards.
\end{abstract}

Instrumental thermoregulatory behavior has been studied in the laboratory under a variety of conditions. First reported in rats (Carlton \& Marks, 1958; Weiss, 1957), thermal reinforcement of instrumental responses has been conducted successfully in molluscs (Downey \& Jahan-Parwar, 1972), reptiles (Regal, 1971), fish (Rozin \& Mayer, 1961), birds (Budgell, 1971; Schmidt \& Rautenberg, 1975), and several mammalian species (Baldwin, 1968; Baldwin \& Ingram, 1967; Carlisle, 1971; Matthews, 1971).

The regulatory efficacy of these responses was demonstrated early by the constancy of body temperature and the close relationship between response rate and parameters of reinforcement (Weiss \& Laties, 1961). For instance, it was consistently found that when rats are barpressing for heat in a cold environment, decreases in reward duration (RD) produce increases in response rate (Baldwin, 1968; Carlisle, 1966; Matthews, 1971; Weiss \& Laties, 1960). This has been attributed to the fact that each reward provides less heat when RD decreases, and consequently the number of responses must increase so that the same amount of heat is obtained. This regulatory interpretation apparently contradicts studies of incentive motivation using food reinforcement, in which the increase in reinforcer magnitude results in increased responding, but may be explained by the fact that the thermal reinforcer usually alters the state of deprivation of

This research was supported by a fellowship from the Brazilian Research Council (No. 201508/83) to R. Refinetti and by NIH Research Grant AM 32984 to H. J. Carlisle. A summary of the results was presented at the Symposium on Thermal Physiology beld at the University of Calgary, Calgary, Alberta, Canada, July 1986. The present address of R. Refinetti is Instituto de Psicologia, Universidade de São Paulo, 05508 São Paulo, Brazil. Requests for reprints should be sent to H. J. Carlisle at the Department of Psychology, University of California, Santa Barbara, CA 93106. the animals. When very short ( $<1 \mathrm{sec}$ ) thermal rewards are used-so that the cold stress is not substantially altered by each reward-response rate increases as $R D$ increases (Leeming, 1968). This incentive effect is also observed when changes in deprivation are prevented by the imposition of a delay between the availability of two consecutive rewards (Matthews, Pinsky, \& Strax, 1974).

The increase in response rate at short RDs under usual experimental conditions suggests the presence of a compensatory behavioral mechanism responsible for the maintenance of constant heat intake. Closer inspection of the experimental data shows, however, that the compensation is not complete. When RD decreases, response rate for irradiated heat does not increase sufficiently to maintain a constant heat intake (Carlisle, 1966; Corbit, 1970; Weiss \& Laties, 1960). This lack of complete compensation has never been examined in detail. The present series of experiments was designed to investigate why heat intake decreases when RD is shortened.

\section{GENERAL METHOD}

Male rats were used in all experiments, except where otherwise stated. They were housed individually at $25^{\circ} \mathrm{C}$ and were fed Purina Laboratory Chow and water ad lib. Each rat's trunk and limbs were shaved twice a week to facilitate learning and performance (Carlisle, 1968).

The experimental chamber, which was slightly adapted for specific experiments, as described below, was a cylindrical wire-mesh cage $(26 \times 20 \mathrm{~cm})$ equipped with a Plexiglas lever $(3 \times 4 \mathrm{~cm})$. Two redbulb infrared lamps were positioned laterally at $45^{\circ}$ to the lever and adjusted to provide an irradiance of $170 \mathrm{~mW} / \mathrm{cm}^{2}$, measured with an Eppley thermopile placed in the center of the cage in front of the lever. Each leverpress response turned the lamps on if they were off, but responses while the lamps were on did not prolong the reinforcement duration. All sessions lasted $60 \mathrm{~min}$ and were conducted in a freezer set to $-9^{\circ} \mathrm{C}$, except where otherwise stated. Responses and rewards were recorded on electronic counters, and 
only data from the last $30 \mathrm{~min}$ of each session were used for analysis. Heat intake was estimated by multiplying number of rewards by $R D$ in each session for each subject. Thus heat intake is expressed as seconds of lamps on. An approximate energetic equivalent (in joules) can be obtained by multiplying the time of lamps on (in seconds) by 34 (Refinetti \& Carlisle, 1986).

\section{EXPERIMENT 1}

The observation that animals work for less heat when $\mathrm{RD}$ is short could be easily explained by ceiling effects related to the subjects or to the experimental apparatus. It is possible that heat intake decreases at short RD because the increase in response rate required to maintain a constant heat intake approaches the rats' physical limit of response emission. Also, since a finite number of rewards can be released per minute, it is possible that reward rate reaches or approaches its limit when $R D$ is short. The present experiment was conducted to investigate these two ceiling effects.

Six albino rats (weighing 290-370 g) were used as subjects. After six training sessions with RD set to $6 \mathrm{sec}$, four test sessions were conducted, each having one of four RDs per session $(2,4,6$, and $8 \mathrm{sec})$. Following these tests, an automatic pellet feeder was attached to the chamber, and the animals (food deprived to $85 \%$ body weight) were trained to barpress for 45-mg Noyes pellets. Four training sessions were conducted at $22^{\circ} \mathrm{C}$ and two test sessions at $-9^{\circ} \mathrm{C}$. The schedule of reinforcement was con- tinuous reinforcement in the heat-reward condition and fixed-ratio 3 in the food-reward condition.

In accordance with results of previous studies (Carlisle, 1966; Corbit, 1970; Weiss \& Laties, 1960), there was a significant decrease in heat intake as $R D$ decreased (Figure 1A) $[F(3,15)=11.32, p<.01]$. This decrease occurred despite a significant increase in reward rate (Figure 1C) $[F(3,15)=36.50, p<.01]$. No evidence was found, however, of a ceiling effect. It can be seen in Figure 1B that the response rates for heat reward were well below the subject ceiling, as estimated by the response rate for food reward at identical conditions of ambient temperature, chamber size, and lever location. Also, Figure 1C shows that the reward rates were well below the time ceiling, as calculated by dividing the number of seconds in a minute by each $R D(2,4,6$, and $8 \mathrm{sec})$. Therefore, ceiling effects are not the cause of the reduction in heat intake at short RDs.

\section{EXPERIMENT 2}

Other methodological artifacts could explain the observation that animals work for less heat when $R D$ is short. If a large number of short rewards provide more absorption of heat than do a small number of long rewards, then the animals might obtain the same amount of heat in both conditions in spite of the fact that the heat lamps stay on for a longer time when RD is longer. To test this hypothe-
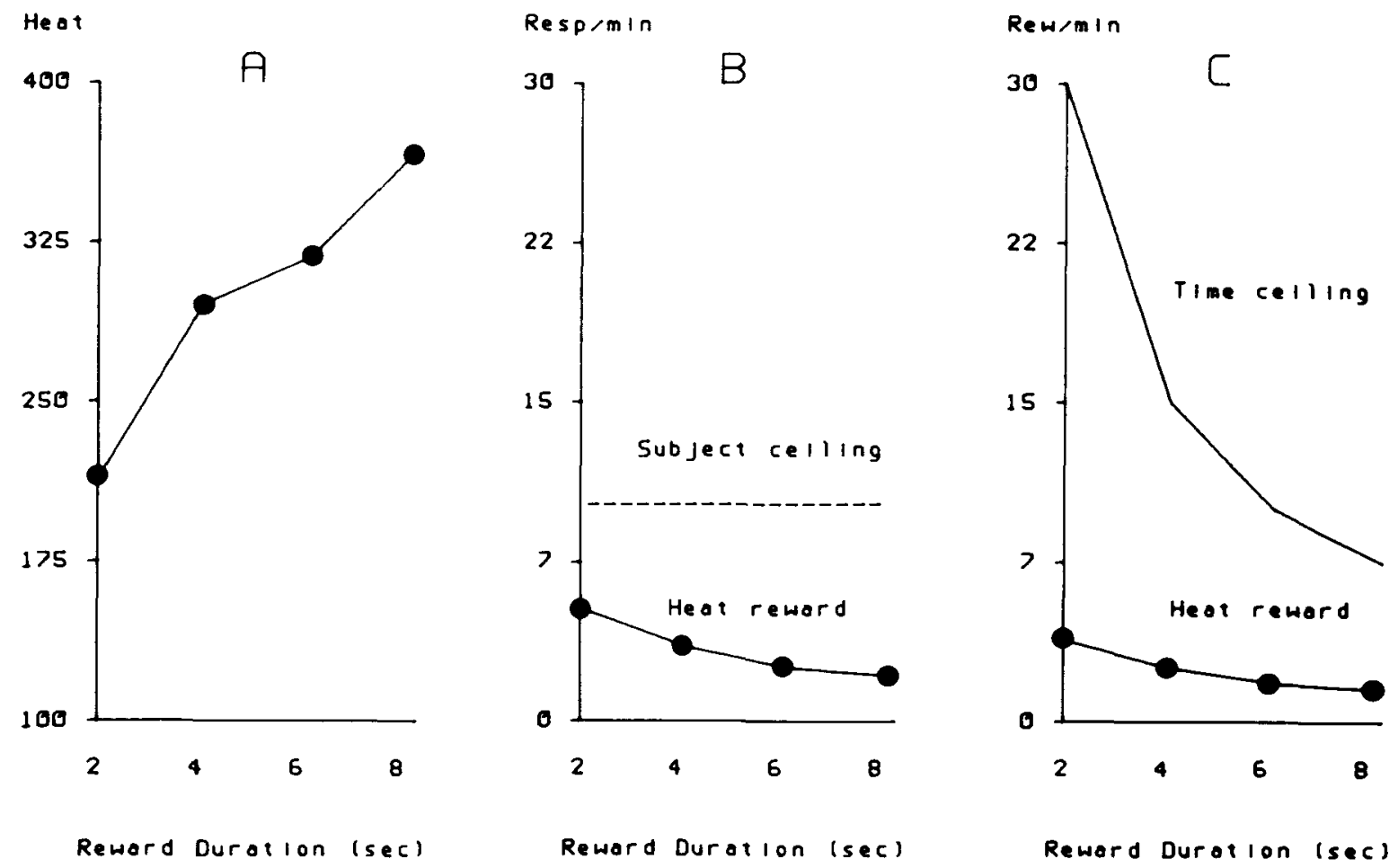

Figure 1. Heat intake (A), response rate (B), and reward rate (C) as functions of reward duration. 


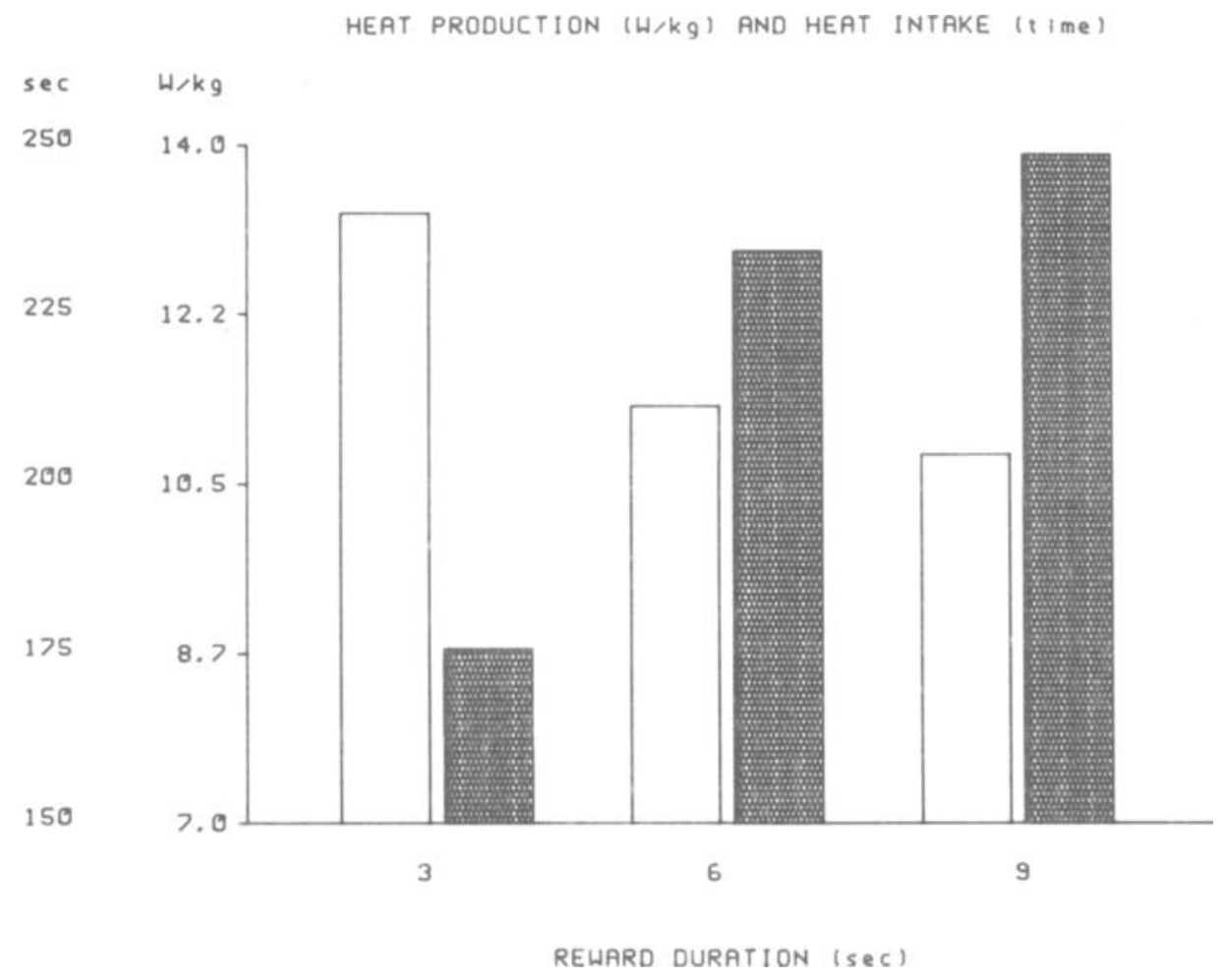

Figure 2. Heat production (white blocks) and heat intake (dark blocks) as functions of reward duration.

sis, we simultaneously measured barpresses and oxygen consumption. If the amount of heat actually absorbed is the same at long and short RDs, then metabolic heat production should also be the same under both conditions. On the other hand, if heat intake decreases at short RD, then heat production should increase to compensate for the smaller intake.

Nine hooded rats (weighing 350-400 g) were used in this experiment. A 4-liter spherical plastic chamber equipped with a Plexiglas lever $(2 \times 3 \mathrm{~cm})$ and air inflow and outflow connectors was substituted for the wire-mesh cage. Air was drawn from the chamber at a rate of 3 liters $/ \mathrm{min}$, and a sample of $250 \mathrm{ml} / \mathrm{min}$ was sent to a Beckman oxygen analyzer. Ambient temperature was $3^{\circ} \mathrm{C}$.

After four training sessions, each animal was tested at RDs of 3,6, and $9 \mathrm{sec}$ (one RD per session). Heat production was calculated from oxygen consumption (corrected to STPD), assuming a mixed-diet respiratory quotient of 0.82 . Rectal temperature $(6 \mathrm{~cm}$ deep) was measured before and after a session with a Yellow Springs Telethermometer (Model 46) and thermistor probe (\#402).

The main results are shown in Figure 2. Heat intake increased significantly as $R D$ increased $[F(2,16)=7.02$, $p<.01$ ]. Heat production, however, decreased as RD increased $[F(2,16)=7.10, p<.01]$. This decrease in heat production was a compensation for the increase in heat intake, since body temperature was not affected by $\mathrm{RD}[F(2,16)=3.08, p>.05]$. It seems, therefore, that if there is a difference in heat absorption at different RDs, it cannot account for the observed difference in heat intake. The data show that the thermoregulatory system proceeds as if there were a difference in heat intake; namely, it modulates heat production to compensate for changes in heat intake.

\section{EXPERIMENT 3}

The reduction in heat intake at short $\mathrm{RD}$ might also be explained by the fact that more responses are required to obtain the same amount of heat when RD is short. In other words, responding at short $\mathrm{RD}$ might involve more effort from the animal, and consequently a reduction in the motivation to respond would be expected under this condition. If this is the case, the inclusion of an additional source of effort should have differential effects on the performance of a rat's barpressing at short and long $\mathrm{RD}$. Thus, we tested animals under different conditions of force required to depress the lever.

Four male and four female hooded rats (weighing 220-340 g) were used as subjects. Tests were conducted in the wire-mesh cage. After 6 training sessions, 12 test sessions were conducted according to a factorial subjects $X$ treatments design, with the subjects as blocks and $\mathrm{RD}$ $(2,4,6$, and $8 \mathrm{sec})$ and force requirement $(0.2,0.6$, and $1.0 \mathrm{~N})$ as treatments.

The results are shown in Figure 3. As expected, heat intake was significantly affected by $\operatorname{RD}[F(3,77)=22.5$, $p<.01]$ and by force requirement $[F(2,77)=24.3$, 
HEAT INTAKE (time)

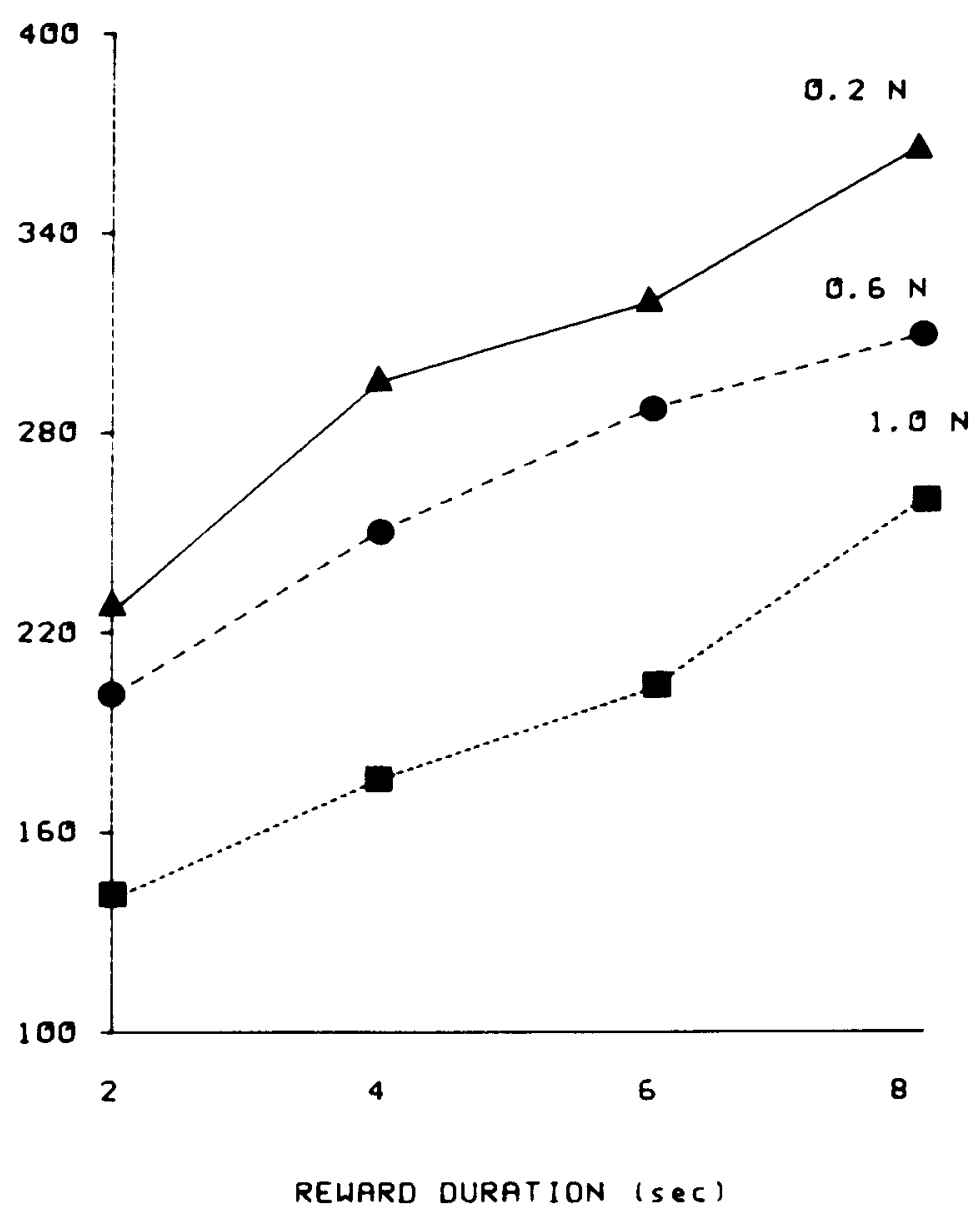

Figure 3. Heat intake as a function of reward duration with response effort as parameter.

$p<.01]$. However, the three curves have very similar slopes, so that the interaction between RD and force requirement is not significant $[F(6,77)=0.78, p>.05]$. If the higher response rate at short RD did imply higher response effort, the animals would be expected to be more sensitive to changes in force requirement at short $R D$ than at long $\mathrm{RD}$. When $\mathrm{RD}$ is long, the animals are exposed to a single source of effort (the resistance of the lever); when $\mathrm{RD}$ is short, they are exposed to two sources of effort (the resistance of the lever and response rate). Because no interaction of RD and force was found, we conclude that the effort hypothesis cannot explain the lower heat intake at short RD.

\section{EXPERIMENT 4}

Visual observation of the animals during Experiment 3 revealed some peculiar characteristics of their operant response. Many animals spent most of the session lean- ing on the lever and emitted a new response by quickly releasing and redepressing the lever. This suggests that the rats never learned the most efficient topography of the response. To obtain a reward, the rat has to press the lever. But if the lever is not released, the lamps will be turned off after a few seconds and will not be turned on again. If the animals do not learn the complete topography (i.e., to depress and then release the lever), they will spend extra time holding down the lever and not maximizing rewards. Because more responses are required at shorter RDs, relatively more time would be wasted when RD is short, and consequently less heat would be obtained. The present experiment was designed as a test of this response-topography hypothesis.

The subjects were the same as those used in Experiment 2. An electronic stopwatch was added to the recording circuit so that the time the lever remained depressed could be recorded. Sessions were conducted at $5^{\circ} \mathrm{C}$. Because the subjects were experienced in barpressing for heat, 


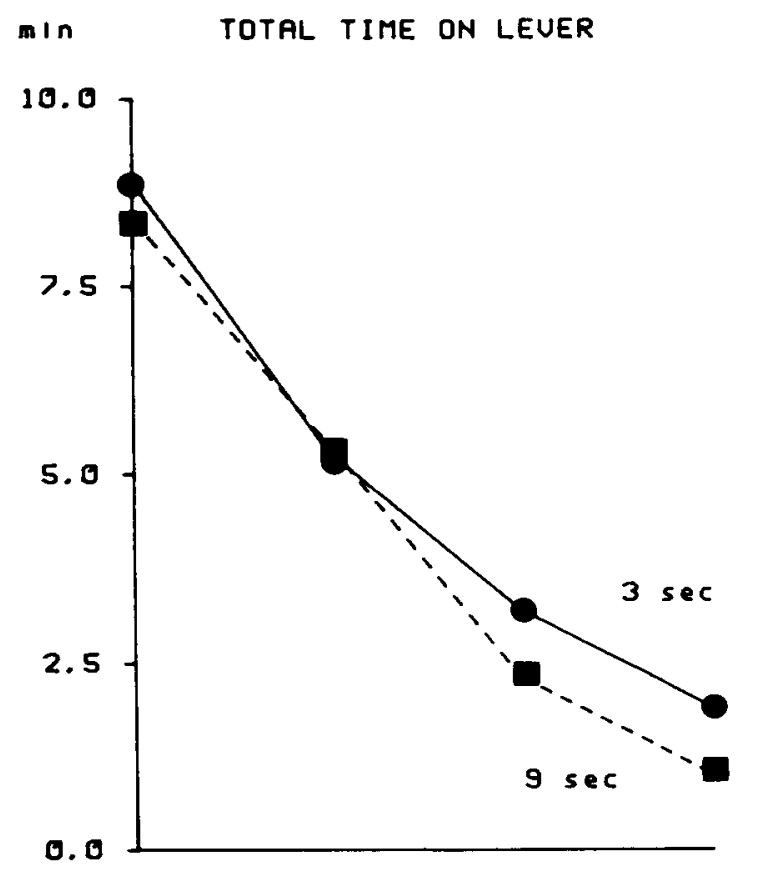

$0.40 .5 \quad 0.6 \quad 0.7$
SEC TIME ON LEUER PER RESPONSE

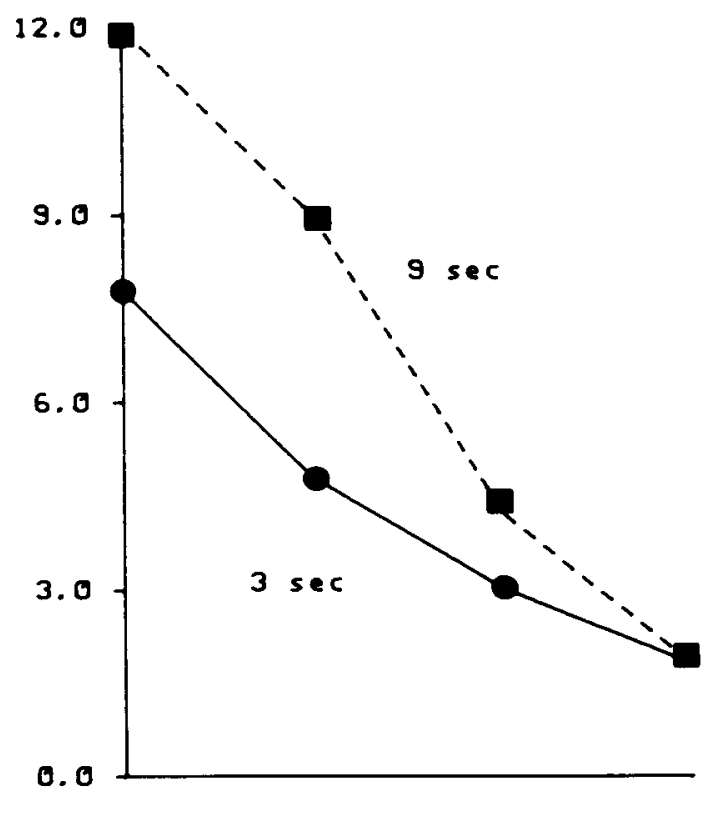

$\begin{array}{llll}0.4 & 0.5 & 0.6 & 0.7\end{array}$

FORCE REQUIREMENT (N)

FORCE REOUIREMENT (N)

Figure 4. Total time the animals spent on the lever during a 30 -min period, and time spent on the lever divided by the number of responses emitted, as functions of response effort with reward duration as parameter.

only one training session was conducted. Test sessions followed a factorial design with RD (3 and $9 \mathrm{sec})$ and force requirement $(0.4,0.5,0.6$, and $0.7 \mathrm{~N})$ as treatments.

The data on time spent on the lever (i.e., time the lever stayed depressed) are shown in Figure 4. Both the absolute amount of time and the time per response (total time on lever divided by number of responses) decreased as force requirement increased $[F(3,56)=9.84, p<.01$, and $F(3,56)=10.5, p<.01$, respectively]. The effect of $R D$ on time per response was also significant $[F(1,56)$ $=9.13, p<.01]$, but the effect on total time was not $[F(1,56)=3.05, p>.05]$.

If the animals held the lever down for only the amount of time necessary to obtain a reward, then the total time spent on the lever should be longer at short RD (because more responses are emitted), the time per response should be independent of RD, and there should be no difference between tests with low or high force requirements. None of these requirements was met. Therefore, an incorrect response topography may be a plausible reason why heat intake decreases when RD is shortened. The next two experiments suggest that this is not the case.

\section{EXPERIMENT 5}

If an incorrect topography is the cause of the anomaly in thermoregulatory behavior, then special training should be able to correct the response and consequently eliminate the anomaly. Thus we compared the performance of rats trained as usual to depress a lever to obtain a warm reward with that of animals trained to release the bar after depressing it. For the latter group, reinforcement was contingent on release of the lever after it had been depressed.

Twelve albino rats (weighing 250-370 g) were used in this experiment. The equipment was set so that the lamps would be turned on for a predetermined amount of time when the lever was either depressed (depress group) or released (release group). Ambient temperature was $-9^{\circ} \mathrm{C}$.

Six rats were assigned to each group and trained to barpress for a 6-sec reward during six sessions. In the test session, RD was $6 \mathrm{sec}$ during the 15-min warm-up period, $3 \mathrm{sec}$ during the following $30 \mathrm{~min}$, and $9 \mathrm{sec}$ during the last $30 \mathrm{~min}$.

The results are shown in Figure 5. Again, RD had a significant effect on the amount of heat obtained by the animals $[F(1,20)=39.72, p<.01]$. However, the special training administered to the release group improved the performance of the animals very little; the effect due to the interaction of group and RD did not reach statistical significance $[F(1,20)=1.05, p>.05]$. Although further improvement might be expected with further training, it is clear that making the reward contingent on the release of the lever did not contribute much to improved 


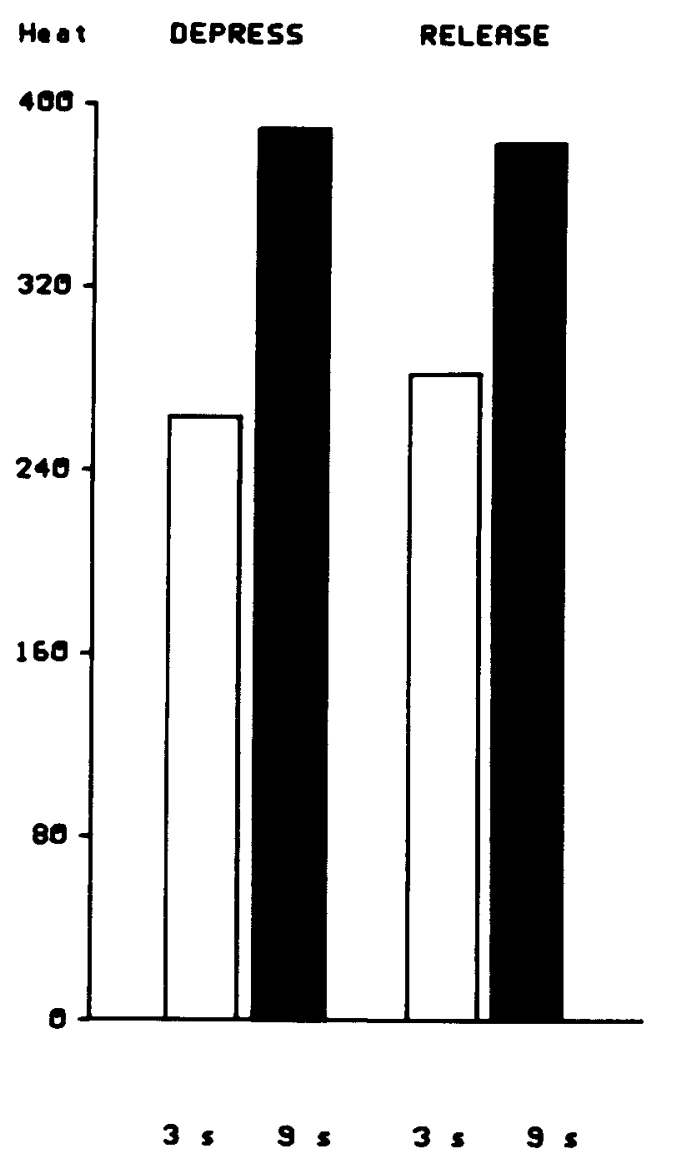

Figure 5. Heat intake at two different reward durations (3 sec and $9 \mathrm{sec})$ for two groups of rats (depress and release).

performance. A more effective training seems to be required to obtain results that clearly support the responsetopography hypothesis.

\section{EXPERIMENT 6}

The results from Experiment 5 showed that leverrelease training only slightly improves the performance of the animals at short RDs. In Experiment 6, we changed the operand so that it required a reaching response which is incompatible with prolonged lever depression.

Six of the rats from Experiment 5 were used as subjects. A Plexiglas key $(3 \times 4 \mathrm{~cm})$ attached to the wall of the cage, $14 \mathrm{~cm}$ above the floor, was substituted for the lever. As usual, the lamps were activated when the subject depressed the key. However, the animal had to stand on its hind legs and extend the body to depress the key, a posture that rats do not maintain for long periods. Because all rats had previous experience with thermal rewards (seven barpress sessions), only three additional training sessions were conducted (with RD set to $6 \mathrm{sec}$ ). Two test sessions were conducted for each subject. In one of them, the 3-sec reward period (30 min) preceded the 9-sec period (30 $\mathrm{min})$; in the other, the order was reversed.

The subjects emitted many more responses than they had emitted in the standard leverpress condition. At reward duration of $9 \mathrm{sec}$, for instance, they pressed the key 150 times in $30 \mathrm{~min}$ but pressed the lever (Experiment 5) only 58 times $[t(5)=5.21, p<.01]$. The amount of heat obtained at this RD was similar in both situations (keypress: 350; leverpress: 390). However, heat intake decreased significantly from 350 at 9 -sec $R D$ to 183 at $3-\sec \operatorname{RD}[t(11)=4.89, p<.01]$. Therefore, precluding the animals' leaning on the operand did not eliminate the decrease in heat intake at short RD. We conclude that the improper response topography is not the cause of lower heat intake at short $\mathbf{R D}$.

\section{EXPERIMENT 7}

In Experiment 2, we showed that the reduced heat intake at short RD is compensated by an elevated metabolic heat production, which means that the central controller of autonomic thermoregulation receives the information concerning the reduction in heat intake. However, it is possible that behavioral regulation depends on a different input of thermal information. Because heat obtained at short RD is more regularly distributed (many short pulses as opposed to few long pulses at long RD), thermal afference from the skin might be enhanced at short RD. This would lead to the observed decrease in behavioral responding. In order to test this hypothesis, we provided the animals with free rewards at the same rate as they had obtained reward when depressing the lever and also allowed them to supplement this free heat by pressing the lever for additional heat.

Six albino rats (weighing 360-440 g) were used as subjects. Four training sessions $(R D=6 \mathrm{sec})$ preceded a test session in which RDs of $3 \mathrm{sec}$ and $9 \mathrm{sec}$ were used. In this test session, heat intake was 270 at $R D=3 \mathrm{sec}$ and 378 at $R D=9 \mathrm{sec}$. Based on these data, the rates of free reward were calculated: 3.0 rewards per minute for $R D=3 \mathrm{sec}$, and 1.4 rewards per minute for $R D=9 \mathrm{sec}$. As a control procedure, a rate of 1.0 reward per minute for $\mathbf{R D}=3 \mathrm{sec}$ was also scheduled. Sessions 6 and 7 for each animal were adaptation sessions to the new schedule of reinforcement: the heat lamps remained on as long as the lever was depressed (ad-lib reinforcement) and not for fixed intervals predetermined by the experimenter. In the next three sessions, free rewards were provided according to the rates described above, and the animals were allowed to supplement the free heat by pressing the lever for additional heat. One free-reward rate was used per session, in a randomized order across subjects.

The results are shown in Figure 6. The black section of each block indicates the amount of heat earned during a 30-min period (mean for 6 subjects). The white section 


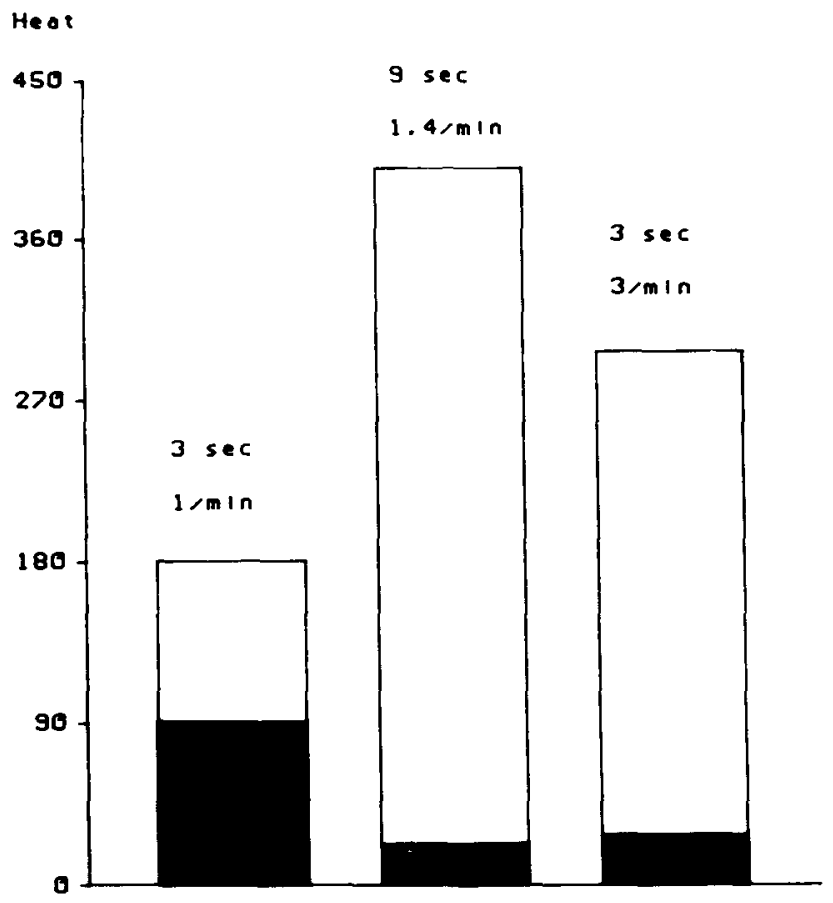

Figure 6. Voluntary heat intake at three different conditions of concomitant free-reward release.

(free heat) is stacked on the black section, so that the total height of each bar indicates the total amount of heat obtained during the session. Although the animals received more free heat at the 9-sec reward condition (middle bar) than at the 3-sec condition (right bar), the earned heat intake in the two conditions did not differ $[t(5)=0.49$, $p>$.10]. This suggests that the two different amounts of free heat produced comparable afferent signals. Thus, in the regular condition of fixed-duration rewards, the amount of heat obtained when $R D=3 \mathrm{sec}$ generated afferent signals equivalent to those generated by the amount of heat obtained when $R D=9 \mathrm{sec}$, even though the two amounts are physically different.

The control condition (left bar) suggests that the total amount of heat required is reduced when it can be earned under ad-lib conditions, perhaps because shorter rewards are selected. In order to evaluate this issue, we conducted additional sessions in which the duration of each barpress was recorded on a printout counter while the animals worked for heat under the ad-lib schedule. Figure 7 shows frequency polygons for three of the rats (1-h sessions). Although there is some intersubject variability, it is clear that most of the heat pulses were relatively short. Especially worth noting is the fact that in the ad-lib schedule the animals have entire control of the duration of each reward. Therefore, the fact that they chose very short rewards more frequently is evidence of a preference for shorter rewards. This supports the hypothesis that many short fixed-duration rewards produce a greater afferent signal than few long rewards.

\section{EXPERIMENT 8}

The main conclusion of the preceding experiments is that heat intake decreases at short reward durations be-
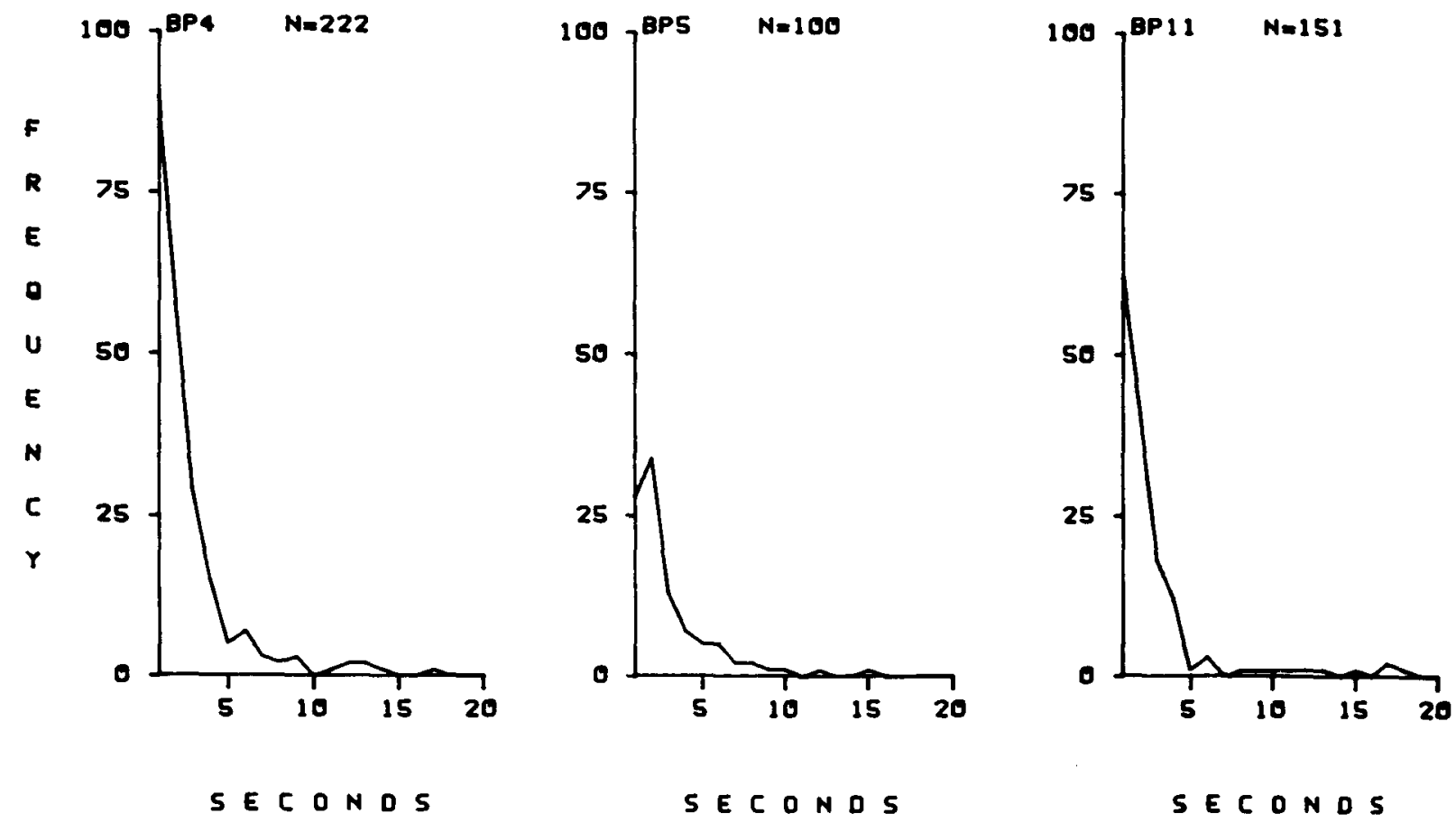

Figure 7. Frequency polygons of response duration under the ad-lib schedule of reinforcement. 
cause of some aspect of the stimulus unrelated to the physical heat content. In the present experiment, we examined the characteristics of the stimulus at the skin.

Skin surface and subcutaneous temperatures were recorded in an anesthetized shaven albino rat. Small copper-constantan thermocouples housed in polyethylene tubing (Clay Adams PE-50) were attached to the skin surface with adhesive tape and to the subcutaneous space through a small incision in the lateral abdominal wall. The thermocouple response was instantaneous for the time scale employed (time constant $=90 \mathrm{msec}$ ). Temperatures were read with a digital meter (Sensortek, Model BAT12) and recorded on a chart recorder (Houston Instrument, 4500 Microscribe). The animal was anesthetized with Nembutal $(60 \mathrm{mg} / \mathrm{kg})$ and placed in the same chamber used for behavioral studies in Experiment 1. Ambient temperature was $-9^{\circ} \mathrm{C}$. Changes in skin and subcutaneous temperature were produced by pulses of infrared irradiation $\left(170 \mathrm{~mW} / \mathrm{cm}^{2}\right)$ of different durations ranging from 1 to $16 \mathrm{sec}$.

Alterations in skin and subcutaneous temperature produced by heat pulses of different durations are shown in Figure 8. Changes in surface temperature (A) were more pronounced and lasted longer than changes in subcutaneous temperature (B). Also, surface temperature dropped rapidly at the termination of the stimulus, whereas the change in subcutaneous temperature persisted as heat absorbed at the skin surface penetrated to subcutaneous tissue. The initial segment of the curves is similar for the different reward durations. Especially at the subcutaneous level, however, the subsequent fall in temperature is much more gradual when RD is long.

Changes in surface skin temperature for 3-sec and 9-sec RDs are plotted in Figure 8C with a better temporal resolution than in Figure 8A (the long tails of the curves were
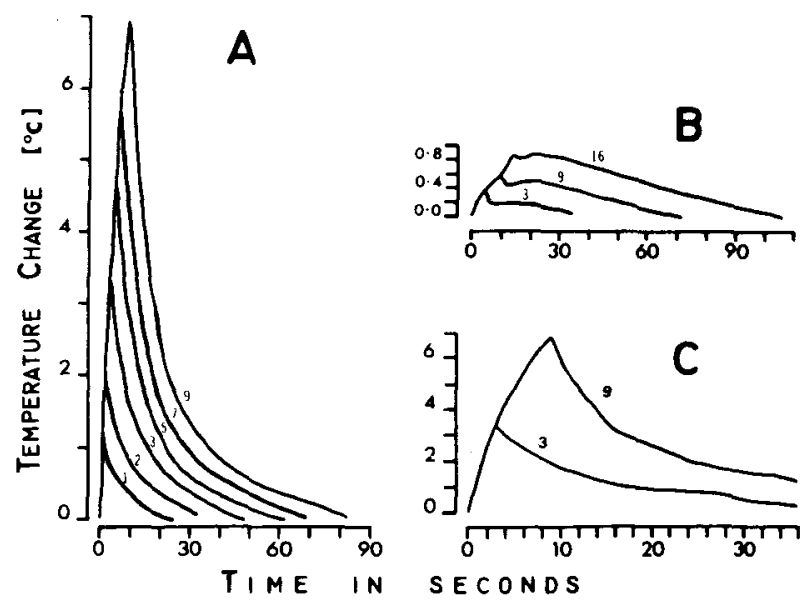

Figure 8. Changes in skin-surface temperature (A) and in subcutaneous temperature (B) produced by pulses of heat of different durations. Skin surface temperature is shown with an expanded time scale for 3-sec and 9-sec pulses in C. omitted). It is evident that changes in skin temperature are not proportional to the amount of irradiated heat. A 9-sec RD contains three times more heat than a 3-sec RD but produces a change in skin temperature that is only two times the change produced by a 3-sec RD. Since thermoreceptors respond to temperature and not to heat (Hensel, 1981), the rats would be expected to work for twice (and not three times) as many rewards when $R D$ is $3 \mathrm{sec}$ than when it is $\mathbf{9} \mathrm{sec}$. Indeed, the rats in Experiments 1-6 obtained an average of 1.9 more rewards (range: 1.6-2.2) when working for 3-sec rewards than when working for 9-sec rewards. Short rewards also generate a much more transient stimulation than do long rewards. This might explain the more intense perception of heat when RD is short, since skin thermoreceptors are known to respond more intensely to transient than to static stimulation (Hensel, 1981). Thus the rate of change in skin temperature (which is greater for more frequent but brief RDs) could explain the decrease in heat intake at short RD.

\section{GENERAL DISCUSSION}

This series of experiments was designed to determine why heat intake during barpressing for heat is lower at short RDs, despite an increase in response rate. Experiment 1 showed that the lower heat intake at short RDs cannot be explained by a subject or time ceiling effect, because the animals have the physical ability to increase response rate, and their rate of obtaining rewards is well below the limits of the apparatus. Experiment 2 showed that the animals compensate for the decrease in heat intake by increasing metabolic heat production. Consequently, the observed decrease in heat intake is not an artifact due to a higher heat absorption at short reward durations that could result in an equally effective heat intake at all reward durations. Experiment 3 showed that the reduction in heat intake cannot be explained by a difference in response effort. It is true that the maintenance of a constant intake of heat requires the emission of more responses at short $\mathrm{RD}$, but this difference in response effort seems to have no significant effect on heat intake. Experiment 4 supported the hypothesis that the decrease in heat intake could be due to an inadequate response topography, namely, the depression of the lever for periods longer than necessary. However, Experiments 5 and 6 failed to provide further support for this hypothesis. The animals do show an inadequate response topography under ordinary leverpress conditions, but this cannot account for the decrease in heat intake.

Experiment 7 provided evidence for the hypothesis that animals work for less heat at short RD because the afferent signals from skin thermoreceptors do not reflect accurately the heat content of the distal stimulus. Apparently, several short rewards produce a greater signal than do fewer long rewards. This does not seem to be directly related to the phenomenon of temporal summation. At 
least in humans, the critical duration for temporal summation in the thermal senses is only 1-3 sec at the threshold level (Eijkman \& Vendrik, 1961; Stevens, Okulicz, \& Marks, 1973), and temporal summation tends to disappear at higher levels of stimulation (Marks \& Stevens, 1972, 1973).

The results of Experiment 8 suggest that the higher efficacy of short rewards is related to the physical characteristics of heat absorption in the skin. First, although a 3-sec reward contains three times less heat than a 9-sec reward, it produces a change in skin-surface temperature that is almost half the change produced by a 9-sec reward. Thus if the animals were working to maintain a constant skin temperature, rather than constant heat intake, we would expect them to work for twice (and not three times) as many rewards when $R D=3 \mathrm{sec}$ as when $R D=9 \mathrm{sec}$, which is exactly what they did. Second, short rewards produce a higher rate of temperature change than long rewards, which is likely to result in a more effective stimulation of skin thermoreceptors. As a consequence, many short pulses are perceived as more intense than few long pulses. Again, response rate should not be in good inverse proportion to $R D$ if the animals respond to conserve skin temperature instead of heat intake.

It should be stressed that although behavior seems to be controlled by skin temperature, the autonomic system responds to the change in the amount of heat and produces compensatory alterations in metabolism (Experiment 2). This is in agreement with previous studies showing that changes in ambient temperature will activate behavioral thermoregulation preferentially to autonomic regulation (Corbit \& Ernits, 1974; Schmidt \& Simon, 1981). Changes in ambient temperature also activate autonomic responses when behavioral efficacy is restricted either overtly or by the choice of reinforcement parameters (Adair, 1976; Ingram, 1973; Refinetti \& Carlisle, 1986).

The interpretation above is a regulatory one in that it emphasizes the contribution to an RD effect of an afferent signal originating from skin thermoreceptors. A nonregulatory contribution to thermal behavior of incentive motivation might seem inappropriate, because response rate increases as reward magnitude decreases. (The opposite result would be expected if heat provided an incentive in the same manner as do appetitive rewards.) However, the fact that more heat is obtained for large as opposed to small rewards is consistent with an incentive interpretation. This would localize the RD effect to a motivational property of the stimulus and not the skin, but it would also necessitate defining incentive in terms of what the animal obtains and not what it does. An incentive effect using rate of response as the measure of thermal incentive has been demonstrated by Matthews et al. (1974), who used convective heating and cooling for both drive and reward conditions and interposed a delay between successive rewards in order to reduce the satiating effect of prior rewards. Under these conditions, the animals worked more for a large than for a small re- ward. Incentive can also be measured in terms of preference, since an animal should prefer the more rewarding of two stimuli. In Experiment 7 of the present study, a clear preference for short as opposed to long rewards was evident when the animals were allowed to control the duration of reinforcement, an observation that is not consistent with an incentive interpretation.

\section{REFERENCES}

ADAIR, E. R. (1976). Autonomic thermoregulation in the squirrel monkey when behavioral regulation is limited. Journal of Applied Physiology, 40, 694-700.

BaLDWIN, B. A. (1968). Behavioural thermoregulation in mice. Physiology \& Behavior, 3, 401-407.

BALDWIN, B. A., INGRAM, D. L. (1967). Behavioural thermoregulation in pigs. Physiology \& Behavior, 2, 15-21.

BuDGelL, P. (1971). Behavioural thermoregulation in the Barbary Dove (Streptopelia risoria). Animal Behaviour, 19, 524-531.

CARusLe, H. J. (1960). Heat intake and hypothalamic temperature during behavioral temperature regulation. Journal of Comparative \& Physiological Psychology, 61, 388-397.

CARIJSLE, H. J. (1968). Initiation of behavioral responding for heat in a cold environment. Physiology \& Behavior, 3, 827-830.

Carussue, H. J. (1971). Behavioral temperature regulation in Cynomolgus and Pig-tailed macaques. Joumal de Physiologie, 63, 226-228.

Carlton, P. L., * Marks, R. A. (1958). Cold exposure and heat reinforced operant behavior. Science, 128, 1344.

CorBrT, J. D. (1970). Behavioral regulation of body temperature. In J. D. Hardy, A. P. Gagge, \& J. A. J. Stolwijk (Eds.), Physiological and behavioral temperature regulation (pp. 777-801). Springfield, MA: Thomas.

CoRBrT, J. D., \& ERNITs, T. (1974). Specific preference for hypothalamic cooling. Journal of Comparative \& Physiological Psychology, 86, 24-27.

Downey, P., Jahan-PArwar, B. (1972). Cooling as a reinforcing stimulus in Aplysia. American Zoologist, 12, 507-512.

Eukman, E., \& Vendrik, A. J. H. (1961). Dynamic behavior of the warmth sense organ. Journal of Experimental Psychology, 62, 403-408.

Hensel, H. (1981). Thermoreception and temperature regulation. London: Academic Press.

INGRAM, D. L. (1973). The efficiency of operant thermoregulatory behaviour in pigs as determined from the rate of oxygen consumption. Pflügers Archiv, 353, 139-149.

LeEMING, F. C. (1968). Response rate as a function of magnitude and schedule of heat reinforcement. Journal of Experimental Psychology, 76, 74-77.

Marks, L. E., \& Stevens, J. C. (1972). Perceived cold and skin temperature as functions of stimulation level and duration. American Journal of Psychology, 85, 407-419.

Marks, L. E., \& Stevens, J. C. (1973). Temporal summation related to the nature of the proximal stimulus for the warmth sense. Perception \& Psychophysics, 14, 570-576.

MATrHews, T. J. (1971). Thermal motivation in the rat. Journal of Comparative \& Physiological Psychology, 74, 240-247.

Matrhews, T. J., Pinsky, S. D., \& Strax, R. (1974). An incentive effect in thermally motivated behavior. Animal Learning \& Behavior, 2, 173-176.

RefinetT, R., \& Carlisie, H. J. (1986). Complementary nature of heat production and heat intake during behavioral thermoregulation in the rat. Behavioral \& Neural Biology, 46, 64-70.

REGAL, P. J. (1971). Long term studies with operant conditioning techniques, of temperature regulation patterns in reptiles. Journal de Physiologie, 63, 403-406.

Rozin, P., MAYER, J. (1961). Thermal reinforcement and thermoregulatory behavior in the goldfish, Carassius auratus. Science, 134, 942-943. 
SCHMIDT, I., RAUTENBERG, W. (1975). Instrumental thermoregulatory behavior in pigeons. Journal of Comparative Physiology, 101, 225-235.

SCHMmT, I., Simon, E. (1981). Behavioral and autonomic cold defense of pigeons modified by central and peripheral thermal stimulation. In Z. Szelényi \& M. Székely (Eds.), Contributions to thermal physiology (pp. 25-27). Budapest: Akadémiai Kiad6.

Stevens, J. C., Okulicz, W. C., Marks, L. E. (1973). Temporal summation at the warmth threshold. Perception \& Psychophysics, 14, 307-312.

Weiss, B. (1957). Thermal behavior of the subnourished and pantothenic- acid-deprived rat. Joumal of Comparative \& Physiological Psychology, 50, $481-485$.

WeIss, B., LAties, V. G. (1960). Magnitude of reinforcement as a variable in thermoregulatory behavior. Joumal of Comparative \& Physiological Psychology, 53, 603-608.

WEISs, B., LATIES, V. G. (1961). Behavioral thermoregulation. Science, 133, $1338-1344$.

(Manuscript received August 12, 1986;

revision accepted for publication November 24, 1986.) 